\title{
OCLUSÃO ARTERIAL RETINIANA PARADOXAL EM PACIENTE COM COMUNICAÇÃO INTERATRIAL
}

\author{
PARADOXAL RETINAL ARTERY OCCLUSION IN PATIENT WITH \\ INTERATRIAL COMMUNICATION
}

Bruna Santana Boaventura ${ }^{1}$, Caio Elisei Neves Machado ${ }^{1}$, Lacy Carvalho Falcão $^{1}$, Giusepe Graciolli. ${ }^{2}$

\begin{abstract}
RESUMO
A oclusão do ramo arterial retiniano (ORAR) é uma emergência oftalmológica caracterizada pela presença de perda aguda da visão com danos devastadores e muitas vezes irrecuperáveis. Esta patologia afeta mais comumente adultos por volta dos 60 anos e raramente atinge pessoas com idade inferior a 30 anos. Os sintomas apresentados variam de acordo com a localização da lesão. O diagnóstico da ORAR é realizado após uma revisão detalhada da clínica do paciente, juntamente com exame oftalmológico, vascular e cardiológico. O tratamento se baseia na tentativa de restabelecer rapidamente o fluxo arterial retiniano. Os tratamentos agudos não invasivos atuais incluem a redução da pressão intraocular, enquanto as terapias invasivas costumam ser trombolíticas. Entre as causas de ORAR, destacam-se as causas cardiológicas, sendo que o forame oval patente (PFO) é uma causa rara, com poucos casos descritos. Este relato de caso retrata um quadro clínico raro, pois trata-se de uma paciente do sexo feminino, jovem, que foi acometida por uma oclusão arterial retiniana secundária a um evento embólico na presença de um PFO. Palavras-chave: Comunicação Interatrial; Forame Oval Patente; Oclusão da Artéria Retiniana.
\end{abstract}

\begin{abstract}
Brazil Brunch retinal artery occlusion (BRAC) is an ophthalmic emergency characterized by acute vision loss with devastating and often irretrievable damage. This pathology commonly affects adults around the age of 60 and rarely affects people under the age of 30 . The symptoms vary according to the location of the lesion. The diagnosis of BRAC is made after a detailed review of the patient's clinic, along with ophthalmic, vascular and cardiological examination. Treatment is based on an attempt to quickly restore retinal arterial flow. Currently acute noninvasive treatments include lowering intraocular pressure, while invasive therapies are often thrombolytic. Among the causes of BRAC, cardiological causes stand out, and the patent foramen ovale (PFO) is a rare cause, with few cases described. This case report portrays a rare clinical picture, as it is a young female patient who was affected by a retinal arterial occlusion secondary to an embolic event in the presence of a PFO.
\end{abstract}

Key-words: Heart Septal Defects, Atrial; Foramen Ovale, Patent; Retinal Artery Occlusion. declararam que não existem conflitos de interesses. 


\section{INTRODUÇÃO}

A primeira vez em que foi relatada a obstrução da artéria central da retina (OACR) foi em 1859 e foi feita por Von Graefe em um paciente com endocardite e múltiplos êmbolos sistêmicos. ${ }^{1}$

A OACR e a oclusão do ramo arterial retiniano (ORAR) são emergências oftalmológicas geralmente caracterizadas pela presença de perda aguda da visão com danos devastadores e irrecuperáveis da mesma. Um estudo experimental realizado com macacos Rhesus demonstrou que, após 240 minutos de isquemia, ocorre danos maciços e irreversíveis ao tecido retiniano. ${ }^{2}$

Esta patologia afeta adultos geralmente por volta dos 60 anos e raramente atinge pessoas com idade inferior a 30 anos. Os homens são mais propensos a serem afetados do que as mulheres. ${ }^{3}$

Geralmente, a OACR ou a ORAR são provocadas por um evento embólico. Sendo que a oclusão do ramo da artéria central da retina representa de 38 a $40 \%$ das oclusões arteriais retinianas. Os sintomas apresentados irão variar de acordo com a localização da lesão. ${ }^{1}$

O diagnóstico da OACR e da ORAR é realizado após uma revisão detalhada da clínica do paciente, juntamente com exame oftalmológico. Além disso, os exames vasculares e cardiológicos também são muito importantes para fornecerem informações sobre o progresso e a etiologia. ${ }^{4}$

Os tratamentos se baseiam na tentativa de restabelecer rapidamente o fluxo arterial retiniano. Na maioria dos casos, a cegueira se instala em razão da ausência de tratamento em tempo capaz para a recuperação da visão. Os tratamentos agudos não invasivos atuais incluem a redução da pressão intraocular, enquanto as terapias invasivas costumam ser trombolíticas. $^{2,4}$

$\mathrm{Na}$ investigação causal, é muito importante dar atenção a eventos cardiológicos que podem ser sugestivos para o acontecimento da OACR e da ORAR. Dentre estes eventos, abordaremos o Forame Oval Patente, o qual representa a cardiopatia do caso relatado.

O forame oval patente (PFO) representa uma condição cardíaca congênita, a qual consiste em uma comunicação interatrial persistente. Esta comunicação permanece devido a uma falha no fechamento do forame oval, normalmente, no primeiro ano de vida, gerando uma potencial derivação da direita para a esquerda. ${ }^{5}$

A associação entre PFO e o evento embólico sistêmico e cerebral tem sido consistentemente apoiada, principalmente em adultos jovens com menos de 55 anos de idade. ${ }^{6} \mathrm{Na}$ ocorrência de um evento isquêmico arterial agudo com a presença de PFO e a ausência de uma fonte embólica clara, deve-se suspeitar de embolia paradoxal, principalmente em pacientes com menos de 60 anos. $^{7}$

A OACR e a ORAR em pacientes jovens é uma entidade clínica rara e poucos casos com PFO sendo a sua causa foram descritos. ${ }^{6}$ Desta forma, este relato de caso retrata um quadro clínico raro, já que se trata de um paciente feminino, jovem, que foi acometido por uma oclusão arterial retiniana paradoxal secundária a um evento embólico na presença de uma PFO.

\section{DESCRIÇÃO DO CASO}

Paciente, sexo feminino, 38 anos, cirurgiã dentista, é admitida no dia 11 de novembro de 2019 com a queixa de "ver mancha em olho direito" há cinco dias, associada a dor retrobulbar e sensação de corpo estranho. Sintomas com início súbito e sem causa evidente, como trauma. Foi encaminhada com suspeita de descolamento de retina seroso.

Realizou-se um exame de fundo de olho o qual demonstrou uma oclusão arterial no terço proximal da arcada temporal superior (ATS), sem a presença de sinais de coriorretinite, no olho direito (OD) da paciente. Em seguida, foi realizada uma angiografia com fluoresceína que revelou área hipofluorescente por bloqueio de contraste ao longo da arcada temporal superior compatível com hemorragia intrarretiniana. Não foram observadas hipofluorescência por defeito de enchimento retiniano (isquemia) ou vazamento de contraste.
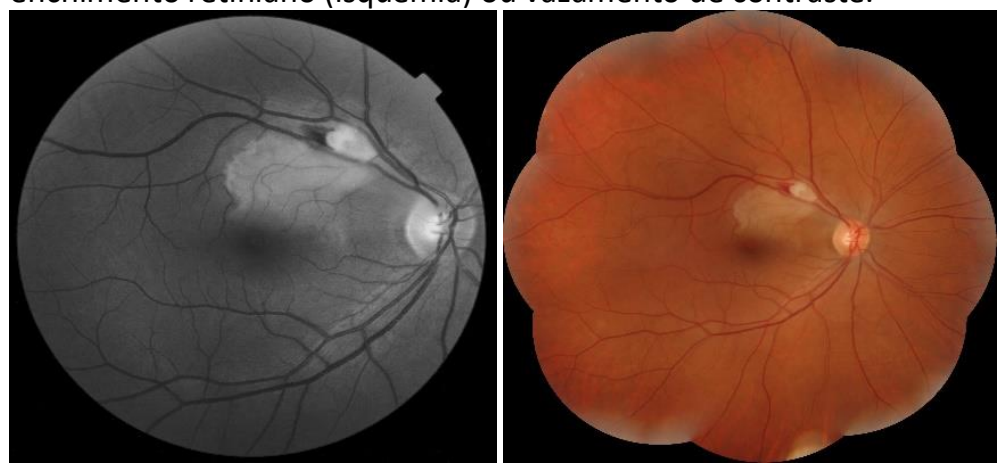

Imagem 2- Retinografia do olho dirkitbegem 3- Retinografia colorida do

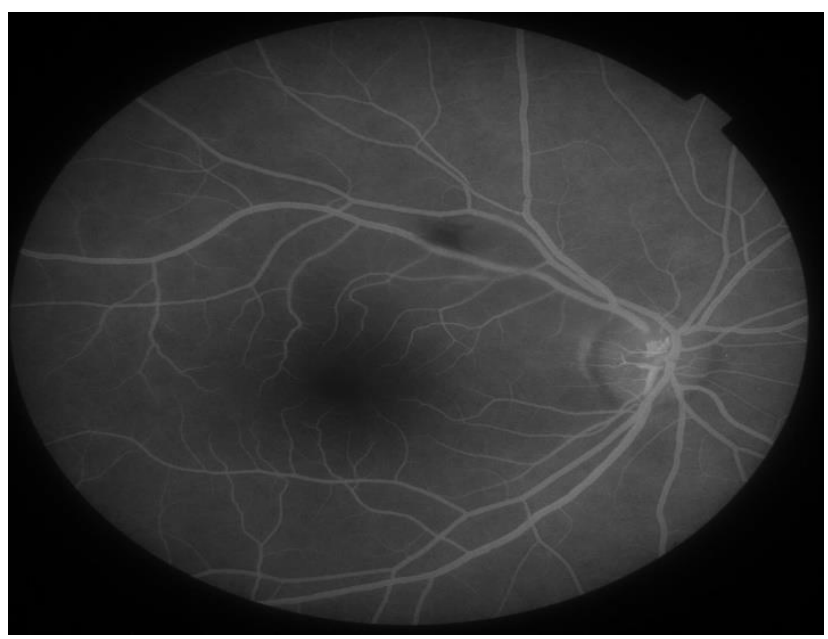

Na sequência, devido há um edema macular pós-oclusão arterial retinana, realizou-se uma campimetria visual (CV) e uma tomografia de coerência óptica (OCT). Na campimetria computadorizada observou-se defeito campimétrico central e 
em pontos para-centrais inferiores do olho direito, compatível com a lesão anatômica. Na OCT foi possível concluir que havia um leve edema intrarretiniano sem formações císticas em região para-foveal nasal superior, com hiperreflectividade óptica nas camadas retinianas internas associadas ao sombreamento óptico em camadas profundas.

Uma investigação clínica, com diversos especialistas, foi realizada para análise da possível causa deste quadro. Em 17 de dezembro de 2019 foi realizado um ecocardiograma no qual revelou um aneurisma de septo interatrial (abaulado para direita), com pequena comunicação de tamanho $2,5 \mathrm{~mm}$, que ao doppler revelou fluxo esquerdo-direito mínimo. Dia 29 de janeiro de 2020 foi realizado um ecocardiograma transesofágico, sob sedação, confirmando o diagnóstico de comunicação interatrial e no qual, após contraste ecocardiográfico por veia periférica, houve passagem de bolhas pelo septo atrial durante a manobra de Valsava. Não foram visibilizados trombos intracavitários. Com estes exames, foi possível diagnosticar a presença de Forame Oval Pérvio (FOP) com shunt direita-esquerda, sendo encaminhado para a correção cirúrgica, com a finalidade de evitar novos eventos trombóticos. Além disso, foi administrado Somalgin $100 \mathrm{mg}$ ao dia para uso contínuo.

Dia 20 de fevereiro de 2020 realizou-se o procedimento cirúrgico percutâneo, por meio da punção da veia femoral, para a oclusão do FOP com prótese Coocon №25. Através da ecocardiografia transesofágica 3D intraoperatória, confirmou o diagnóstico previamente citado. Após o implante da prótese, realizado com sucesso, observou-se resolução completa do shunt (sem shunt residual ao ecocardiograma transesofágico). No dia 13 de maio de 2020 a paciente retornou para exame oftalmológico. Sua acuidade visual foi de $20 / 60$ em olho direito e 20/20 em olho esquerdo, na fundoscopia revelou mácula com reflexo alterado em olho direito, sua biomicroscopia foi normal ao AO em ambos os olhos e a pressão intraocular foi $12 / 12$ $\mathrm{mmHg}$. Desta forma, foram solicitados angioflurosceinografia retiniana e campimetria visual a cada 6 meses para seguimento. A paciente segue fazendo uso de Somalgin $100 \mathrm{mg}$ ao dia.

\section{DISCUSSÃO}

A oclusão do ramo arterial retiniano (ORAR) é uma emergência oftalmológica rara em pacientes jovens, apresentando maior incidência por volta dos 60 anos. Quando presente em pacientes com idade inferior a 45 anos costuma estar associada a anormalidades sistêmicas ou cardíacas subjacentes. . $^{8,9}$

No caso clínico supracitado, a paciente de 38 anos foi diagnosticada com PFO após a realização de um ecocardiograma transesofágico. O PFO é a anormalidade cardíaca congênita mais comum, presente em 10 a 35\% da população. A maior parte dos pacientes é assintomática e não apresenta complicações no decorrer da vida. Entretanto, diversos estudos mostram uma forte associação entre a PFO e o risco de eventos embólicos, como a embolia paradoxal (EP). ${ }^{8,9}$ A EP ocorre pela passagem de um êmbolo da circulação venosa para a circulação sistêmica. Esse diagnóstico deve ser suspeitado em pacientes com PFO que apresentaram um evento isquêmico agudo sem uma fonte embólica clara, o que se enquadro no caso clínico apresentado.,10

Em relação à conduta terapêutica em pacientes com PFO que apresentaram eventos isquêmicos, há duas modalidades de tratamento disponíveis: o uso de antiagregantes plaquetários e o fechamento do forame por cateterismo com colocação de próteses. Não há consenso em relação à superioridade de qualquer um dos métodos. ${ }^{11} \mathrm{~A}$ paciente do caso realizou o fechamento do forame por cateterismo e seguiu com o uso contínuo do antiagregante plaquetário Somalgin $100 \mathrm{mg}$ ao dia. O diagnóstico da ORAR é realizado comumente por meio do quadro clínico, do exame do fundo de olho e da angioflurosceinografia retiniana. Classicamente, a ORAR apresenta-se com perda súbita e profunda da visão, de forma indolor, altitudinal ou setorial do campo visual. ${ }^{12}$ No caso clínico, a paciente apresentava dor retrobulbar e sensação de corpo estranho, o que diferia do quadro clássico.

Na ORAR, o exame de fundo de olho apresenta: estreitamento das artérias e veias com enlameamento e segmentação da coluna sanguínea, retina branca edematosa correspondente à área de isquemia. Podem ser observados um ou mais êmbolos. A AF mostra atraso no enchimento arterial e hipofluorescência do segmento envolvido devido ao bloqueio da fluorescência de fundo pelo edema retiniano. ${ }^{12}$ Os exames de fundo de olho e a AR realizados no caso clínico apresentavam achados compatíveis com ORAR.

Segundo Kanski (2012), o tratamento da oclusão arterial retiniana aguda pode ser tentado em oclusões de duração menor que 24 a 48 horas na apresentação. Após esse período, a perda visual torna-se irreversível devido ao desenvolvimento do infarto retiniano. ${ }^{12}$ No caso clínico apresentado, a paciente procurou atendimento oftalmológico após 5 dias do início dos sintomas, o que configura perda visual irreversível.

Os exames oftalmológicos realizados cerca de 6 meses após o início do quadro revelaram redução de acuidade visual e a presença de alterações na mácula durante a fundoscopia. Isto ocorre devido a obstrução permanente da artéria afetada, o que é observada como uma área atenuada. Em alguns casos, entretanto, pode ocorrer recanalização da artéria obstruída, deixando sinais sutis ou ausentes na fundoscopia. ${ }^{12}$

\section{CONCLUSÃO}

A OACR e a ORAR são eventos raros em pacientes jovens e femininos. Desta forma, é de extrema importância uma minuciosa avaliação sistêmica para a identificação da etiologia deste quadro e, assim, evitar novos eventos isquêmicos em outros órgãos ou tecidos. Além disso, a identificação e o tratamento precoce da oclusão arterial retiniana é fundamental para evitar perdas irreversíveis da visão.

\section{REFERÊNCIAS BIBLIOGRÁFICAS}

1. Freitas LG; Isaac DL; Gabriel LA; Bianchi LC; Ávila MP. Oclusão de ramo arterial retiniano bilateral. Revista brasileira de oftalmologia. [Internet. Agosto de 2013. 72(4):271-73. Disponível em:_https://doi.org/10.1590/S003472802013000400013

2. Domingues CG; Capitanio CF; Abreu LB; Mattana MV. Oclusão da artéria central da retina em paciente com poliangeíte microscópica. Revista brasileira de oftalmologia. 
[Internet]. Dezembro de 2015. 74 (6): 386-89. Disponível em: http://dx.doi.org/10.5935/0034-7280.20150081

3. Tavares PR; Oliveira MR; Diniz EC; Agostini RM. Oclusão de artéria central da retina associada ao forame oval patente. Revista brasileira de oftalmologia. [Internet]. Fevereiro de 2014. 73 (5): 308-10. Disponível em: http://dx.doi.org/10.5935/0034-7280.20140065

4. Nakayama LF; Bergamo VC; Silva LC; Morais NS. Avaliação de paciente com oclusão de artéria central da retina com artéria cilioretiniana patente através de angiografia por tomografia de coerência óptica (OCT-A): Um relato de caso. Arquivos brasileiros de oftalmologia. [Internet]. Fevereiro de 2018. 81(3):242-6. Disponivel em: http://dx.doi.org/10.5935/0004-2749.20180048

5. Sheth HG; Laverde-Koning T; Raina J. Undiagnosed Patent Foramen Ovale Presenting as Retinal Artery Occlusion - An Emerging Association. Journal of Ophthalmology. [Internet]. 11 de setembro de 2009. Volume 2009, ID 248269. Disponível em: https://doi.org/10.1155/2009/248269

6. Clifford L; Sievers R; Salmon A; Newsom RS. Central retinal artery occlusion: association with patent foramen ovale. Eye. [Internet]. Junho de 2006. 20(6):736-8. Disponível em: https://doi.org/10.1038/sj.eye.6701983

7. Chaikof EL; Campbell BE; Smith RB. Paradoxical embolism and acute arterial occlusion: rare or unsuspected?. Journal of vascular surgery. [Internet]. Setembro de 1994; 20 (3): 377-84. Disponível em: https://doi.org/10.1016/07415214(94)90135-X

8. Ho IV, Spaide R. Central retinal artery occlusion associated with a patent foramen ovale. Retina. [Internet]. Fevereiro de 2007. 27(2):259-60. Disponível em: https://doi.org/10.1097/iae.0b013e318030cc24

9. Mohamed $Q$, Ormerod O, Downes SM. Retinal artery obstruction, migraine and patent foramen ovale. British journal of ophthalmology. [Internet]. Novembro de 2006. 90(11):1432. Disponível em: http://dx.doi.org/10.1136/bjo.2006.093617.

10. Magalhães E; Torreão J; Costa N; Freitas N; Melo A. Papel do forame oval patente e da válvula de Eustáquio nos eventos tromboenbólicos. Arquivos de Neuro-Psiquiatria. [Internet]. Junho de 2006. 64(2): 245-48. Disponível em: http://dx.doi.org/10.1590/S0004-282X2006000200014

11. KANSKI JJ; BOWLING B. Córnea. Oftalmologia Clínica: uma abordagem sistêmica.7 ed; 2012.559-66 pag. 